Relations industrielles

Industrial Relations

\title{
Précis de l'arbitrage des griefs, par Fernand Morin et Rodrigue Blouin, Les Presses de l'Université Laval, 1980, 507 pp.
}

\section{Jean-Louis Dubé}

Volume 36, numéro 2, 1981

URI : https://id.erudit.org/iderudit/029165ar

DOI : https://doi.org/10.7202/029165ar

Aller au sommaire du numéro

Éditeur(s)

Département des relations industrielles de l'Université Laval

ISSN

0034-379X (imprimé)

1703-8138 (numérique)

Découvrir la revue

Citer ce compte rendu

Dubé, J.-L. (1981). Compte rendu de [Précis de l'arbitrage des griefs, par Fernand Morin et Rodrigue Blouin, Les Presses de l’Université Laval, 1980, 507 pp.] Relations industrielles / Industrial Relations, 36(2), 449-451.

https://doi.org/10.7202/029165ar

Tous droits réservés @ C Département des relations industrielles de l'Université Laval, 1981
Ce document est protégé par la loi sur le droit d'auteur. L’utilisation des services d'Érudit (y compris la reproduction) est assujettie à sa politique d'utilisation que vous pouvez consulter en ligne.

https://apropos.erudit.org/fr/usagers/politique-dutilisation/ 


\section{RECENSIONS BOOK REVIEWS}

Précis de l'arbitrage des griefs, par Fernand Morin et Rodrigue Blouin, Les Presses de l'Université Laval, 1980, 507 pp.

En 1975, Fernand Morin et Rodrigue Blouin avaient publié un ouvrage intitulé L'arbitrage des griefs au Québec. Comme ils le soulignent dans leur préambule, les auteurs ont été obligés d'effectuer une refonte complète du livre, surtout en raison des nombreuses modifications et additions apportées en 1977 aux dispositions du Code du travail concernant l'arbitrage des griefs. Considérant en plus les nombreuses décisions judiciaires et arbitrales rendues depuis 1975, une foule de sujets qui n'avaient été qu'effleurés à cette date et traités avec ampleur dans le présent volume, nous nous rendons compte qu'il s'agit tout simplement d'un nouvel ouvrage.

Le premier titre situe l'arbitrage des griefs dans le cadre des principales règles du droit des rapports collectifs de travail; il souligne le particularisme de cette justice par rapport à celle des tribunaux judiciaires et dresse un tableau de l'évolution historique du processus de l'arbitrage des griefs depuis 1944.

Ensuite, les auteurs présentent et analysent succinctement les principales règles de droit applicables à l'arbitrage des griefs celles du Code du travail, celles élaborées par les parties dans leur convention collective et leur explicitation et interprétation par les arbitres et les tribunaux judiciaires. Les pratiques sont ajoutées comme autre source de droit. On trouve des propos fort intéressants au sujet de l'utilisation de la jurisprudence et de la portée de la règle du stare decisis.

Le titre III traite des différentes sortes de griefs et des parties à l'arbitrage. Ayant établi la distinction entre les conflits d'intérêts et les conflits de droit en relations de travail, les auteurs situent les griefs parmi ces derniers. Après un bref exposé sur le grief au sens strict, c'est-à-dire celui correspondant à la définition du Code du travail, le grief assimilé par voie conventionnelle est examiné en discutant de l'étendue et des limites de la liberté des parties à cet égard. Puis, on fait une étude des différents types de griefs: individuels, collectifs et de principe. Enfin, une section est consacrée aux griefs assimilés par voie législative, tel celui concernant le retour à l'emploi du gréviste ou du salarié affecté par un lockout et celui prévu aux articles 124 et suivants de la Loi sur les normes de travail. On examine ensuite d'une part la règle à l'effet que le syndicat accrédité est en principe le titulaire du grief et d'autre part le droit d'intervention du salarié.

Le titre IV est intitulé «Le tribunal d'arbitrage: ses coordonnées générales». On y traite de plusieurs sujets parmi lesquels nous ne mentionnerons que les principaux.

Le titre VIII, intitulé «Le contrôle de l'application de la convention collective», regroupe des sujets très variés. Ainsi, on fait une étude fort intéressante du pouvoir réparateur du tribunal d'arbitrage en discutant la question des droits de la direction et en illustrant avec beaucoup d'exemples. Suivant des développements sur la distinction entre mesure disciplinaire et mesure administrative et sur l'énoncé de principe «obéir d'abord, se plaindre ensuite». Enfin le lecteur trouve un très bon exposé sur les difficultés nombreuses de cerner ce pouvoir réparateur de l'arbitre.

Le titre IX contient des propos très pertinents sur le processus décisionnel du tribunal 
d'arbitrage et le pouvoir de contrôle des tribunaux judiciaires.

Enfin, le dernier titre traite des régimes spéciaux d'arbitrage, tel celui de l'industrie de la construction, et donne un aperçu des systèmes d'arbitrage en vigueur dans les entreprises de juridiction fédérale, en Ontario, en Colombie-Britannique et aux États-Unis.

Cet ouvrage contient aussi des études sur plusieurs sujets que nous n'avons pas mentionnés.

Il est indéniable que cet ouvrage comble une lacune importante qu'il y avait depuis quelques années en ce domaine au Québec. Mentionnant que le tribunal d'arbitrage doit procéder selon le système contradictoire et non inquisitoire, on précise qu'il n'a pas cependant un rôle passif au cours de l'enquête. Une énumération des objections préliminaires habituelles précède une analyse du moment opportun pour les parties de les soulever et pour l'arbitre de les trancher. Puis les auteurs font l'ébauche d'une théorie générale selon laquelle l'employeur aurait souvent le fardeau de la preuve en raison du fait que c'est lui qui prend les initiatives et que de son côté, le syndicat est confiné à un rôle le plus souvent simplement contestataire. Suivent alors des exposés sur les questions suivantes: la qualité de la preuve, les procédés de preuve et les plaidoiries.

Au sujet des règles d'interprétation des conventions collectives, les auteurs soulignent avec beaucoup de pertinence l'absence d'uniformité parmi les arbitres et celle d'un ouvrage de synthèse sur la question. Après avoir mentionné la nécessité d'avoir des règles d'interprétation, les auteurs soulignent leur diversité et leur incertitude. Bien souvent cependant ces règles ne sont que de la logique et du bon sens, et n'on rien de sacré. Dès lors, l'arbitre peut utiliser entre autres les règles d'interprétation des lois et des contrats mais doit se garder d'avoir une attitude servile à leur égard en raison du particularisme de la convention collective. Par la suite, les auteurs analysent sous douze rubriques plusieurs règles usuelles d'interprétation et donnent quelques illustrations.
La règle de l'impartialité du titulaire d'un pouvoir quasi-judiciaire est examinée en soulignant les nuances particulières concernant le tribunal d'arbitrage. Les questions des différents types de tribunal et la nomination de leur(s) membre(s) sont présentées. Analysant le problème de l'objet de l'arbitrage, les auteurs traitent du pouvoir du tribunal d'arbitrage de décider de sa propre juridiction et ajoutent des précisions sur l'étendue et les limites de cette dernière.

Après avoir donné une série d'exemples de procédures internes de réclamation, les auteurs analysent de façon originale et très intéressante la question de l'effet juridique du défaut par les parties de respecter ces procédures. Rappelant les célèbres décisions de la Cour Suprême du Canada à ce sujet, ils soulignent les conséquences démesurées qu'en ont tirées les tribunaux et les arbitres. On trouve une très bonne analyse critique de la légalité de ces dispositions de convention collective en les confrontant avec le principe du droit à l'arbitrage que les syndicats ont reçu en échange d'une renonciation à la grève pendant la durée d'une convention collective. Enfin, on procède à une analyse cle la question de la prescription d'un grief.

Le titre sixième est intitulé "Enquête et preuve». Les auteurs traitent du pouvoir de l'arbitre d'établir les règles de procédure, de la question de l'amendement d'un grief et de la requête pour détails. Les ouvrages de Palmer (Collective Agreement Arbitration in Canada) et de Brown \& Beatty (Canadian Labour Arbitration) pouvaient et peuvent toujours servir, mais le particularisme du système juridique québécois imposait un livre pour le Québec. Certains pourraient être tentés de reprocher aux auteurs de ne pas avoir fait un dépouillement et une analyse systématique de la jurisprudence arbitrale québécoise, mais ce serait fort inapproprié. Il n'était de toute évidence pas dans leur intention de faire un équivalent québécois des ouvrages de Palmer et de Brown \& Beatty. Bien que ce dernier pourrait être extrêmement utile, l'approche de Morin et Blouin est différente, en est une de pédagogie, de synthèse et d'analyse des questions de fond souvent très personnel- 
le et originale. Il faut remercier les auteurs de nous faire partager ce fruit de leurs réflexions et de leur expérience.

\section{Jean-Louis DUBÉ}

Université de Sherbrooke

La suspension du contrat de travail, par JeanMarc Béraud, Paris, Éditions Sirey, 1980, 294 pp.

Ouvrage étonnant et remarquable! Étonnant de par l'audace subtile de l'auteur qui s'écarte des explications classiques pour leur substituer une nouvelle conception de la suspension du contrat de travail. Remarquable en raison de l'analyse systématique et alerte qui est présentée sur les concepts et techniques juridiques.

Pour M. Jean-Marc Béraud, ce n'est ni la théorie de l'exception d'inexécution, ni le phénomène de la mutilation unilatérale des effets du contrat non plus d'ailleurs que la notion de l'impossibilité d'exécution par suite de force majeure temporaire qui peuvent servir de base à une approche globale de la suspension du contrat de travail.

Car il s'agit pour l'auteur de rendre compte de la somme totale des manifestations du concept de la suspension dans le cadre du contrat de travail par l'étude du phénomène in se, abstraction faite en un premier temps des causes qui l'engendrent ou des objectifs recherchés. Deux critères sont alors retenus: le maintien de l'existence du contrat de travail; la disparition du lien de subordination. Ces premières constatations permettent à $\mathbf{M}$. J.-M. Béraud de proposer la définition suivante de la suspension du contrat de travail: le droit de l'une ou l'autre des parties d'invoquer la disparition du lien de subordination juridique dans le cadre d'un contrat de travail en cours pour obtenir ou justifier l'arrêt du travail. Il s'agit indéniablement là d'une vision de la suspension en elle-même, d'une approche qui distingue le phénomène juridique de ses causes et finalités.

On notera par ailleurs l'introduction de la notion de la dualité de la suspension du contrat de travail. Il s'agit de l'opposition entre les suspensions qui s'inscrivent dans le déroulement normal du contrat de travail et celles qui se présentent comme des perturbations au déroulement normal. Les premières, dites périodiques ou intrinsèques, englobent les cas des pauses-repas, congés payés, etc., tandis que les secondes, dites accidentelles ou extrinsèques, regroupent les cas d'absence pour cause de maladie, la grève, etc.

Poursuivant sa démarche, l'auteur en arrive à un énoncé à double volet qui alimentera la controverse: pour le salarié, la suspension du contrat de travail se rattache pour lui au droit des personnes et non pas au droit des biens; l'employeur n'engage pas sa personne mais ses biens. L'absence de règle juridique pouvant rendre opérationnelle cette distinction n'a certes par échappé à M. J.-M. Béraud. Il était cependant en droit de réclamer pour le salarié un meilleur respect de sa dignité humaine, ce qui ne saurait être assuré dans le cadre du droit des biens où le travailleur est en définitive traité comme un objet de location.

Suit une étude des effets de la suspension qui est notamment l'occasion pour rappeler que ceux-ci varient selon que l'on considère les obligations discontinues ou les obligations continues dont ont convenu les parties au contrat de travail. L'effort fait pour clarifier les situations vaut d'être signalé. Des développements intéressants sont consacrés à l'influence de la suspension du contrat de travail sur les institutions représentatives du personnel et des syndicats ainsi que sur le pouvoir disciplinaire de l'employeur.

Finalement, les derniers propos portent sur les conflits de suspension et sur la durée. On y trouve là aussi l'intention de clarifier. On sera surpris de la rigueur de l'affirmation voulant que la suspension apparue chronologiquement la première régit, jusqu'à sa disparition, l'ensemble de la période de suspension.

En définitive, comme l'a noté M. Jean Pélissier dans la préface à l'ouvrage, désor- 